\title{
A Semiotic Comparison of Mass Media Representations of the Swine Flu and Covid-19 Pandemics: Observing Narcissus Narcosis
}

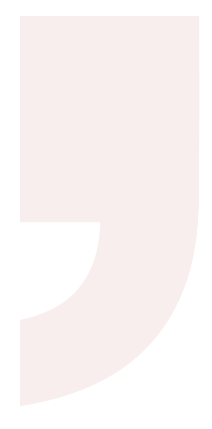

ARTICLE INFO:

Volume: 07

Issue: 01

Summer 2021

ISSN: 2459-2943

DOI: $10.18680 /$ hss.2021.0004

Pages: 45-65

Lic.: CC BY-NC-ND 4.0

KEYWORDS:

Infodemic

Narcissus narcosis

Semantic networks

Topic modeling

Iconicity

\section{ABSTRACT}

$\square$ his paper compares the coverage of the H1N1 and 1 Covid-19 pandemics in ten prominent US daily newspapers. We selected articles that reference disease-specific keywords, published in the period between the declaration of a Public Health Emergency of International Concern by the World Health Organization and the first peak in laboratory-confirmed cases in the USA (20550 articles on Covid-19 and 1705 articles on H1N1). We analyzed the dataset via topic models and semantic networks, which, in a semiotic approach, are understood as iconic models. As the Covid-19 virus produced the first global pandemic in the age of social media, this comparative analysis illustrates how the news media changed the mediasphere in general. During the H1N1 pandemic (2009-2010), newly emerging social media were not mainstream, having a limited impact compared to 2020 at the outbreak of Covid-19. By 2020, social media have definingly changed the mediasphere. Given their affordance for the virulent transmission of media products, the rise of social media stirred the relativization of knowledge and mistrust towards traditional authority and legacy media. Paradoxically, this both democratizes public debate and opens opportunities for misinformation. In this context, the Covid-19 pandemic has been accompanied by a global infodemic, with adverse impact on global health. While the two pandemics are very different, comparing media representations in their early stages, when the viral spread was unpredictable, offers an insight into how 
the emergence of social media impacted traditional newspapers' approach to events of global concern. The analysis reveals that ideological commitments are expressed through the same correlation of topics in both corpora but that, overall, the discourses have different structures. We argue that the remarkable stability of ideological discourses displays what McLuhan termed Narcissus narcosis, namely the numbness experienced socially during media changes.

\section{Introduction}

This paper compares media representations of the Covid-19 and swine flu pandemics in American newspapers through a combination of topic modeling and network analysis. In this way, we observe the similarities and differences in a discourse before and after social media has become the leading news source. These may pass unnoticed, as media changes are difficult to perceive from within. We consider data in newspaper content to reflect on how the rise of social media, not just mere digitalization, alters journalism. In the public discourse on the present Covid-19 pandemic, the 1919 Spanish flu has emerged as the singular historical reference point for a global public health emergency otherwise considered as unprecedented. Such diagnostics face the problem that, even under the theoretical assumption of perfect knowledge in the hands of an "ideal chronicler," the historical significance which, in the future, will be attributed to an event cannot be determined in the present (Danto 2007). The extent of historical precedence, in other words, is not fixed but co-evolves as an event unfolds in time. In respect to the pandemic, up until some point during the year 2020, Covid-19 was not (yet) an event that required observers to look back to 1919 to identify a historical precedent or analogy. Instead, during the first few months when the SARS-CoV-2 virus started spreading worldwide, a range of reasonably comparable cases existed, the most relevant being the 2009 H1N1 (swine flu) pandemic. As the Covid-19 pandemic unfolded in subsequent months, its accumulating effects in terms of public health, governmental responses, as well as social and economic impacts, gradually reduced the plausibility of H1N1, as well as other viral crises (such as the 1968 Hong Kong flu, the 1977 Russian flu or the 2006 avian flu) that have been recurring throughout the world for decades, being relevant historical cases.

And yet, the owl of Minerva always spreads its wings at dusk. Up until a few months into the pandemic, Covid-19 might just as well have been a historically limited-impact event, comparable with the three influenza crises referred to above, rather than the transformational, once-in-a-generation type of event, as it turned out to be. In its early stages, Covid-19 did not display the signs of such a significant and global pandemic. We became aware of that only later and in retrospect. 
A Semiotic Comparison of Mass Media Representations of the Swine Flu and Covid-19 Pandemics: Observing Narcissus Narcosis

Here, we focus on the unfolding of the Covid-19 pandemic in the media, specifically in US-American newspapers, by comparing it with the media coverage of the swine flu pandemic. We ask, to what extent did the public discourse show similarities or differences to such crises during the early months when the historical significance of the Covid-19 pandemic was arguably much more indeterminate than it is today? And what are the broader theoretical implications that we can derive based on identifying such empirical similarities and differences?

Comparing the media coverages of swine flu and Covid-19 pandemics is useful not only because they had a comparable early impact. A particular insight arises from the media change that unfurled in the decade between these events. By the end of 2009, $42 \%$ of US adults reported using at least one social media site; by 2019, 72\% did (Pew Research Center 2021). In brief, by the time of the swine flu outbreak, social media were just emerging, whereas by the time of Covid-19, these constituted the primary source of news in the US (see Mitchel and Shearer 2021). In the US, social media outpaced newspapers as the primary news source in 2018 (Shearer 2018). The transition from all traditional broadcasting media to digital spaces "caused huge disruptions in the traditional news industry, especially the print news industry" (Shearer 2021), as $86 \%$ of US adults report consuming news via a digital device. This medial change is reflected in a semiotic change as the syntax of social media evolves. In 2009 Facebook launched the 'like' button, enabling users to express their appreciation for a post. However, by 2020, the users have at their disposal a code consisting of seven buttons for expressing an emotional response to a post. The need for better differentiation of instantaneous expressions of emotion signals that posting in some new media has become a common practice. In light of such considerations, we compare the coverage of a pandemic before and after social media's rise to dominance in the media environment. The audience behavior and networking on digital social media are quite different from previous media (including digital). Researchers began to notice this in the early 2010s (e.g., Mills 2012; Marwick and boyd 2012; Litt 2012; Litt and Hargittai 2016). In McLuhan's terms (1994: 18), social media are now more impactful in shaping "sense ratios" and "patterns of perception" than traditional broadcasting media, which was not the case in 2009. A first and most crucial observed difference is that the Covid-19 pandemic, the first pandemic of the Digital Age proper, is wrapped in an infodemic (see Cinelli et al. 2020; Hua and Shaw 2020).

\section{Models: topic, semantic, iconic}

We start with an exploratory and open-ended comparative analysis of the US news media reporting on Covid-19 and the 2009 swine flu during the early months of either pandemic. There is extensive literature on infectious disease reporting in the news 
media, including the rapidly growing number of studies concerning Covid-19. Still, we use a relatively novel hybrid method to identify and analyze discursive structures, which we define in terms of linked semantic elements. In analyzing two corpora, comprising articles from large US dailies, we first estimate two Correlated Topic Models that identify the latent semantic structure of news reporting on Covid-19 and the swine flu. Second, we use these models to generate semantic networks, where nodes are topics and arcs represent inter-topic correlations (Rabitz et al. 2021). The resulting models are iconic in semiotic terminology (Kralemann and Lattmann 2013) since they represent their objects through similarity to bring some of the objects' features to the fore. The approach we adopt follows a semiotic conceptualization of digital humanities modeling (Ciula and Eide 2017: i34), where modeling is defined as "a creative process of thinking and reasoning where meaning is made and negotiated through the creation and manipulation of external representations." Adopting such a pragmatic lens, we apply various network-analytic tools and measurements that illustrate remarkable similarities in the two discursive structures.

We interpret our data models in light of McLuhan's classic media theory, particularly adopting his view on media change. On the one hand, McLuhan (1994: 16, 92-93) considered that an essential feature of the "electronic age" is that, unlike previous historical media changes, the contemporary individual can observe the change and, thus, can become aware of some effects of the newly emerging media technology. In this situation, the old and the new medium are both visible, contrasting each other. The social implications of past media changes, such as those produced by the alphabet or printing press, occurred too slowly to be acknowledged individually. If, until the emergence of electronic media, a human individual would populate one mediatic space throughout a lifetime, currently, experiencing several media spaces throughout a lifetime is inevitable.

On the other hand, while nowadays, it is evident that human societies are undergoing a media change (digitalization), the full effects of this change are not easily perceived from within the changing societies. This is due to the nature of media, to the fact that they constitute the lens through which societies perceive and shape themselves. On a more general level, as per Danto's (2007) argument, we cannot determine the historical significance of an unfolding event.

McLuhan (1994: 42-43) also argued that media changes cause a type of numbness. Comparing media discourses on the swine flu and the Covid-19 pandemics offers insight into the specific media change brought about by digitalization. Our argument rests on observing a certain degree of iconicity between the models stemming from the two corpora. The two models share certain structural similarities that are not immediately visible by comparing the corpora. The models we operate with are iconic; namely, their representational quality is based on "a similarity relation be- 
A Semiotic Comparison of Mass Media Representations of the Swine Flu and Covid-19 Pandemics: Observing Narcissus Narcosis

tween themselves and their objects" (Kralemann and Lattmann 2013: 3998), that is, the two corpora. ${ }^{1}$ Instead of comparing the corpora, we compare the models, which give direct access to the characteristics of the corpora in which we are interested. The iconicity of topic models is a little-explored semiotic quality. As both models are iconic signs, the features of the objects they exhibit are easy to compare.

In the spirit of Kralemann and Lattmann's iconic modeling theory, we adopt Charles S. Peirce's (see CP 4.561n²) notion of iconicity, explained simply by Stjernfelt (2007: 49) as "the sign-relation making one phenomenon signify another by similarity in some respects." While similarity can be a relatively trivial criterion for modeling, iconicity is defined "by the direct observation of [an icon] other truths concerning its object can be discovered than those which suffice to determine its construction" (CP 2.279). Genosko (2012: 6) agrees that the defining feature of icons, in the Peircean sense, is "discovery, concept generation and experimentation based on observation." Stjernfelt (2015: 37) clarifies that "[t]his surplus of information is accessed via manipulation of or experimentation with the icon - actions realizing deductive inferences." Adopting this perspective, we deductively infer our models to reflect on newspapers' representations of pandemics of global concern before and after the social media revolution (in this regard, see Mitchel and Shearer 2020; Pew Research Center 2021). Notably, we relate our deductions to Marshall McLuhan's concept of Narcissus narcosis (1994: 55).

Our analysis revolves around the observation that ideological commitments are expressed through the same correlation of topics in both pandemics but that, overall, the discourses have different structures. This means that the newspapers' coverage of the two pandemics is similar, while political, social, and media contexts differ. The remarkable stability of correlations between ideology and topics before and after the mainstreaming of social media accounts, even if only in part, for what McLuhan termed Narcissus narcosis (1994: 55), namely the numbness experienced socially by the extension of the human body consisting in the emergence of new media technologies. The stability of the relation between ideological commitments and topics enduring in an old medium, while more contemporary media have become mainstream and determined changes of discourse, is a "counter-irritant," which "produces a generalized numbness or shock that declines recognition" (McLuhan 1994: 43). Of course, as McLuhan's myth-inspired concept is philosophical, what we observe in empirical data is only an indication of such numbness. In all of its complexity, the idea of Narcissus narcosis is more encompassing and, at the same time, elusive than an empirical dataset can capture.

\footnotetext{
${ }^{1}$ Some of us started developing such an uptake of semantic networks in a semiotic key in Olteanu et al. (2019), where we investigated the structure of semantic networks of environmental sciences.
} 
Nevertheless, we claim that the comparison of models of the datasets brings to light some evidence for the narcosis that accompanies media changes. Primarily, McLuhan's Narcissus narcosis serves as the guiding abduction for this study. In a Peircean view of science (CP 1.240-242), the Narcissus narcosis concept here serves as the abductive (intuitive) stage of an inquiry which we take to coenoscopic (deductive) and idioscopic (inductive) stages by the observation of data that, respectively, are analyzed in a media semiotic lens. As such, we are led to infer back on the phenomenological reality described by what McLuhan termed Narcissus narcosis.

This exploration also finds justification in more recent social semiotics, according to which changes in social environments imply changes in the design (form-content articulation) of messages (Kress 2010: 50). Digitalization must have impacted the content of newspapers in specific ways. At the same time, aligned with McLuhan's theory, social semiotics also observes that "past ways of doing things may be 'hardwired' in technologies" (van Leeuwen 2001: 52). For the consumer of newspapers, hence, the stability of the ideology-topic correlation may give the impression that not much has changed socially, that newspapers remain the accountable source of news they have traditionally been. As van Leeuwen (2001: 52-53) adds, resistance to semiotic change is also exercised "because people with a vested interest in past ways of doing things see their traditional values threatened and try to hold back change," an attitude which is ideologically embodied in technology. While the choice between newspapers or social media as a news source is ideological, newspapers are becoming integrated into digital networks and thus indistinguishable from social media (see Hong 2012; Canter 2013; Ju et al. 2014). This is why, as McLuhan further clarified the Narcissus narcosis concept, "Self-amputation forbids self-recognition" (1994: 43). In brief, the old medium is extended into and transformed by the new one without the consumers' awareness of media products.

In the spirit of van Leeuwen's (2005) and Kress' (2010) social semiotic theory, we offer insight into digitalization by reflecting on the resistance and adaptability to change of newspapers during the unfurling of digitalization semiotic change. This means considering how changes of form, as afforded by mediality, correlate with meaning changes(semantic content, in the case of newspapers). This leads to observing possible new resources for meaning-making that new media are making available for a previously existing medium. This hybridization of the newspaper is also, arguably, a cause for much confusion surrounding the pandemic. Canter (2013: 472) remarks the scholarly "concerns that the wealth of information online is detrimental to journalism." Pressure to compete with social media results in need to produce quantitatively more content and, at times, compromise journalistic inquiry for commercial interests, rendering the newspaper a less reliable medium. In the case of pandemics, this situation might fuel the dramatization of news reporting. 
A Semiotic Comparison of Mass Media Representations of the

\section{Infectious diseases, news media and the case for semantic networks}

Viral pandemics, towards the top of the list of threats to global health security, are part of a larger pandemonium of infectious diseases that have long preoccupied human imagination and, consequently, the mass media. In the past, scholars prioritized the analysis of legacy media, typically via single case studies (Saguy and Almeling 2008; Vasterman and Ruigrok 2013; Raupp 2014; Klemm et al. 2016; Sell et al. 2016) or comparative research designs (Blakely 2003; Wallis and Nerlich 2005; Ungar 2008). In the context of Covid-19, the respective discussions among scholars and policy-makers increasingly include the role of social media and, interconnectedly, of disinformation, propaganda, and "infodemics" (e.g., Au et al. 2020; Bright et al. 2020; Cinelli et al. 2020; Hua and Shaw 2020). Social media are not necessarily less reliable than legacy media, although their comparatively lower transparency and public accountability standards make them more vulnerable to disinformation. More importantly, for this paper, what characterizes social media is the virality implied by the networks they constitute (see Marwick and boyd 2012; Mills 2012; Litt 2012). Virality describes the flow of information through social media networks, not the content and conveyed information (and its truth value). The unique response that the current pandemic received can be partly explained by considering the particular media context (Merchant and Lurie 2020) within which the pandemic is unfolding. While socio-technical change is driving a broader shift in research priorities, from the big-picture perspective, several key themes stand out in the literature on infectious diseases in the media.

First, the media play a crucial role in the social amplification of risk. For instance, Klemm et al. (2016) highlight the part of the mass media in the "dramatizing" of the swine flu pandemic. For the alleged obesity epidemic, Saguy and Almeling (2008) similarly emphasize media dramatization, with Boero (2007) referring to the production of moral panic. Krishnatray and Gadekar (2014) and Goodall et al. (2012) raise similar points regarding the swine flu coverage in, respectively, the Indian and US news media. The related theme of media-induced "fear culture" has been analyzed for Covid-19 in the UK and China (Chaiuk and Dunaievska 2020). Vasterman and Ruigrok (2013) identify "alarmism" in the Dutch media response to the swine flu.

Second, this existence of risk amplification, moral panic, alarmism, or fear culture coincides with the media's crucial role in the indirect management of infectious disease risks. Particularly instructive here is the finding of Ungar (1998), who, studying Ebola in the former Republic of Zaire, finds that media framing shifts from alarmism to management-oriented pragmatism once moral panic poses a plausible public disorder threat. This management-centric media orientation also extends to the cognitive ordering of infectious disease threats. This can happen, for instance, by situating public 
health impacts and policy responses within broader political and economic contexts (Raupp 2014). It can also manifest itself through the supply of heuristics that simplify the socio-cognitive processing of complex and multidimensional public health threats. This notably includes blame attribution through geographical markers (Blakely 2003) or framing morbidity in terms of individual responsibility (Saguy and Almeling 2008).

Alarmism is enhanced by social media, given the viral flow of information on social networking sites. This is clearly illustrated through how an infodemic accompanies the Covid-19 pandemic. After more than a year into this pandemic, whether the stringency and scope of response measures are proportionate to the objective epidemiological profile of SARS-CoV-2 is still a subject of controversy in the media (e.g., Andersen 2020; Cinelli et al. 2020). Cinelli et al. (2020: 1) explain:

The case of the COVID-19 epidemic shows the critical impact of this new information environment. The information spreading can strongly influence people's behavior and alter the effectiveness of the countermeasures deployed by governments. To this respect, models to forecast virus spreading are starting to account for the behavioral response of the population with respect to public health interventions and the communication dynamics behind content consumption.

Objective, medical differences between the two pandemics explain the different public reactions only to a limited extent. Particularly in the initial stages, the evolution of either pandemic was unpredictable, which caused some confusion among the public. While both the swine flu and the Covid-19 pandemics have been the target of attempts to disinform, only the latter is accompanied and made worse by a full-blown infodemic (Cinelli et al. 2020; Hua and Shaw 2020). The fact that it is only in the recent pandemic that misinformation had such momentum testifies to the role that digitalization plays in the formation of an infodemic. While, on the one hand, social media allowed for the spread of misinformation about Covid-19, on the other, it also facilitated the prompt campaigning of expert bodies, such as, first of all, the World Health Organization (WHO 2020b, c; 2021). Remarkably, the WHO (2021) strategy to tackle misinformation is focused on social media platforms, proposing tactics such as using designated hashtags to denounce false information. This confirms Genosko's (2012: 14) observation that the digital network "is inseparable from deception: deceivers are consubstantial with senders and receivers." As the official and traditional authority on health, the WHO has the difficult task of vouching for information by maintaining its authorship clear in an intrinsically virulent network.

The WHO (2020a) stressed the uniqueness of the ongoing pandemic, having explained that "COVID-19 has been the most urgent test of national capacities to 
A Semiotic Comparison of Mass Media Representations of the Swine Flu and Covid-19 Pandemics: Observing Narcissus Narcosis

respond to a health emergency in more than a century." In the early stages of the pandemic, the rate at which the virus spread and its consequences were unpredictable in all areas of human life. Paradoxically, both effective response measures for limiting infection, on the one hand, and confusion and indecision, on the other, are due to the current unprecedented high accessibility to information that new media technologies entail (Merchant, Lurie 2020).

Affording new ways of communication and social organization, the new media reshape the public- and media-sphere in their entirety (e.g., Stjernfelt and Lauritzen 2020: 201-202), while traditional media (e.g., newspapers, radio, TV) have to adapt to the new, content-creating publics (Canter 2013; Ju et al. 2014). Besides the harm caused by disseminating factually wrong information, the coronavirus infodemic leads to a generalized suspicion towards information conveyed by traditionally trusted sources. In this situation, the overall impact of the Covid-19 pandemic on public health globally is boosted, as the overexposure to both correct and wrong media content about the new virus creates confusion and uncertainty. This adds to the strain on mental health, which the pandemic and consequent restrictions already affected (Garfin et al. 2020). In turn, the implications on public mental health have a toll on public opinions about the pandemic. At the same time, digital technology and communication networks offer new opportunities and ways of tackling a crisis that proves critical. Besides responsibly disseminating essential information promptly by expert bodies (e.g., WHO), digital technology makes it possible to keep areas of the economy and society active while strict physical distancing measures are observed.

For this reason, Merchant and Lurie (2020) consider that social media reveal the possibility of enabling a culture of preparedness for crises. Indeed, the WHO has been using its official communication channels (which includes social media) to promptly disseminate information about the pandemic and, also, to counter disinformation with a global outreach. At the same time, we also note that the WHO's responses to the swine flu and Covid-19 pandemics raise questions related to transparency, accountability, and potential conflicts of interest.

\section{Methods and data}

Our data consists of articles published (as of 2020) in the ten largest US daily newspapers, respectively referencing the keywords 'H1N1' / 'swine flu' or 'coronavirus' / 'COVID-19' / 'SARS-CoV-2.' We chose a common time frame during which, arguably, the two pandemics possessed a significant degree of comparability: from the day after the declaration of a Public Health Emergency of International Concern (respectively April 25, 2009, and January 30,2020) by the World Health Organization until the date of the first peak 
in laboratory-confirmed cases in the US (respectively June 14, 2009, and April 11, 2020). This yields 20.550 articles for Covid-19 and 1.705 for the swine flu.

We then proceeded in three steps. First, we preprocessed the two corpora to reduce the overall amount of noise in the data: we concatenated key expressions (such as 'New York' or 'swine flu') to treat them as single, rather than separate terms; normalized acronyms (for treating expressions such as 'CDC' and 'Centers for Disease Control' identically); and removed common stopwords (such as 'the,' 'and,' 'or,' as well as some corpus-specific terms). We removed all special symbols and punctuation and applied a lemmatizer to remove inflections (e.g., transforming all instances of 'took' or 'taking' into 'take').

Second, we estimated two separate topic models. These are a class of statistical methods that identifies 'topics' as clusters of words that are semantically related. Topic models display two types of associations: between topics and terms (e.g., a topic that broadly covers vaccine development might have strong associations with terms such as 'AstraZeneca,' 'mRNA,' 'clinical' and 'trials') and between topics and documents, that is, the extent to which any given document in a corpus is composed of different topics. In semiotic terms, then, a topic model is an iconic model that represents a corpus by highlighting its inner semantic associations between topics and terms, and topics and documents. The similarity between the model and the corpus consists of these associations, which are rendered more easily perceivable and, hence, operative by stripping the model of the corpus's other semantic and syntactic elements. As such, topic models generally enable the discovery of a "low-dimensional latent structure that can explain high-dimensional data" (Roberts et al. 2016: 57). They are already in use in the emerging literature on Covid-19 in the media (e.g., Jo et al. 2020; Quandt et al. 2020) and have been used in media analysis more broadly, for instance, for the identification of frames (Klebanov et al. 2008; DiMaggio et al. 2013). As the details of model estimation are highly technical, we here simply note that we use a standard procedure for identifying a narrow range of viable topic numbers before estimating several hundred separate models within that range. Finally, we retain only those that perform best on key indicators (semantic coherence and exclusivity). We then use the resulting models for gauging the overall semantic content of each corpus. This also allows for observing the role of political ideologies in the framing of discourse: to what extent are the conservative-liberal orientations of the various newspapers in our corpus associated with variation in document-topic loadings? In other words, do conservative and liberal newspapers differ in their emphasis on different topics, and to what extent do such differences replicate between our two cases?

Third, we use our topic models for generating topic networks, which increases the degree of iconicity, as pragmatic to our aim. As texts are mixtures of topics, topic models can provide intertopical correlations of the sort: if text A is about topic X, 
it is also likely to be about topic Y. The possibility of transforming such correlations into networks is often mentioned in the literature yet rarely applied in practice. Topic networks, where topics are nodes and edges are correlations, represent the discursive structure behind a given body of text. They lend themselves towards standard network-analytic methods. Here, we apply network analysis at three levels: node-level centralities (i.e., how 'important' or 'influential' are particular topics within the network?), community structure (i.e., which topics form larger semantic clusters), and network centralization (i.e., how strongly is the network dominated by a single, or a few, topics?). We apply this method from the vantage point of comparing the discursive structures of news media reporting on Covid-19 and the swine flu.

\section{Results}

The two topic models summarize the broader semantic content of the two corpora (coverage of the H1N1 and, respectively, the Covid-19 pandemic) and the relative share of a given corpus that each topic accounts for in figure 1. There and below, to summarize the content of each topic, we provide the three top terms associated

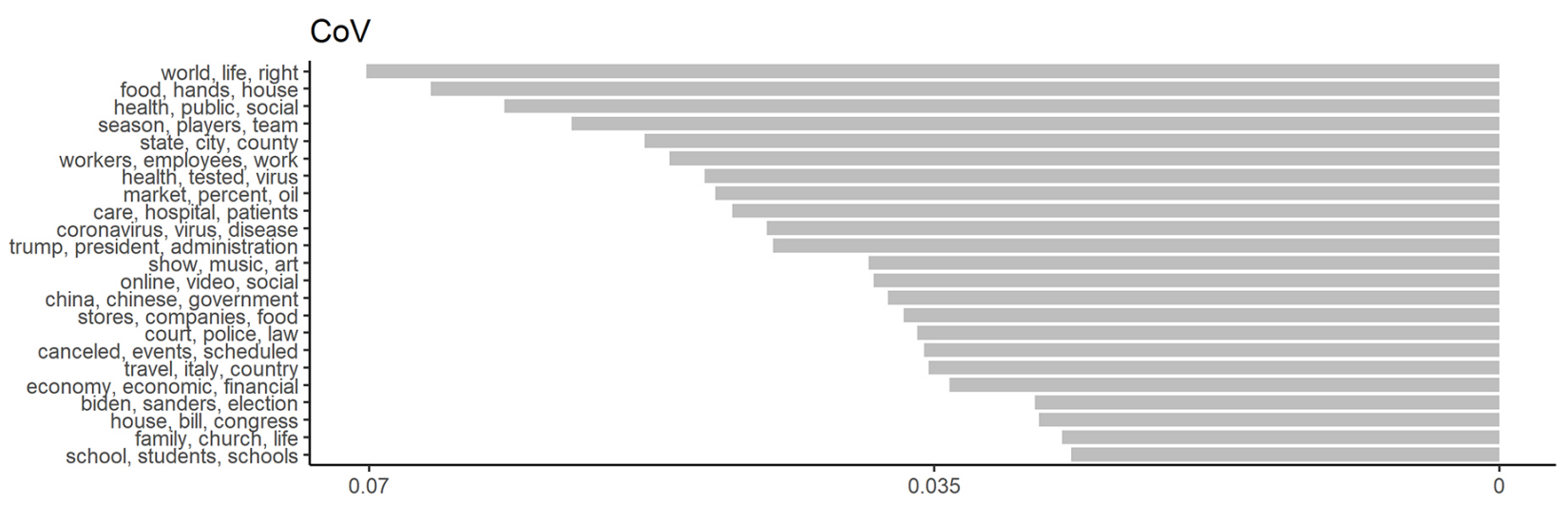

$\mathrm{H} 1 \mathrm{~N} 1$

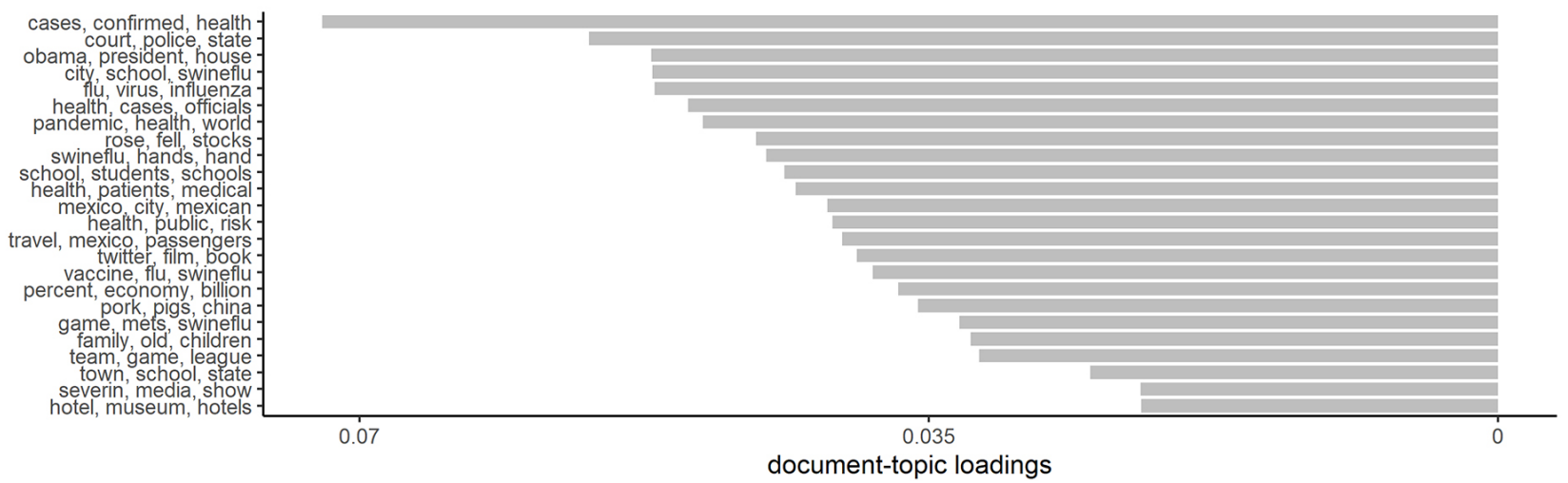

Figure 1. 
with each. For instance, in the case of our swine flu model, the topic 'travel, Mexico, passengers' covers the entry of (possibly or actually) infected persons from Mexico (the epidemiological origin of the swine flu pandemic) into the US territory. For our Covid-19 model, the topic 'Biden, Sanders, election' addresses the pandemic in the context of the 2020 US Presidential elections and the democratic primaries.

We also find that differences in topic weightings between liberal and conservative newspapers are relatively stable across cases (see figure 2). For Covid-19 and the swine flu, we find that conservative newspapers place a significantly stronger emphasis on the economic aspects of the respective pandemic. This effect is mainly driven by our inclusion of articles from the Wall Street Journal and its specific focus on economics and monetary policy. The overall stability in terms of left-right effects on document-topic loadings is remarkable. It indicates that, for the newspapers under consideration, the increases in affective polarization and partisan sorting, which various studies have identified across the US polity, are absent at the level of media reporting.

We now turn to network analysis. For each corpus, we generated an undirected and weighted network of topics and intertopical correlations. Figure 3 visualizes

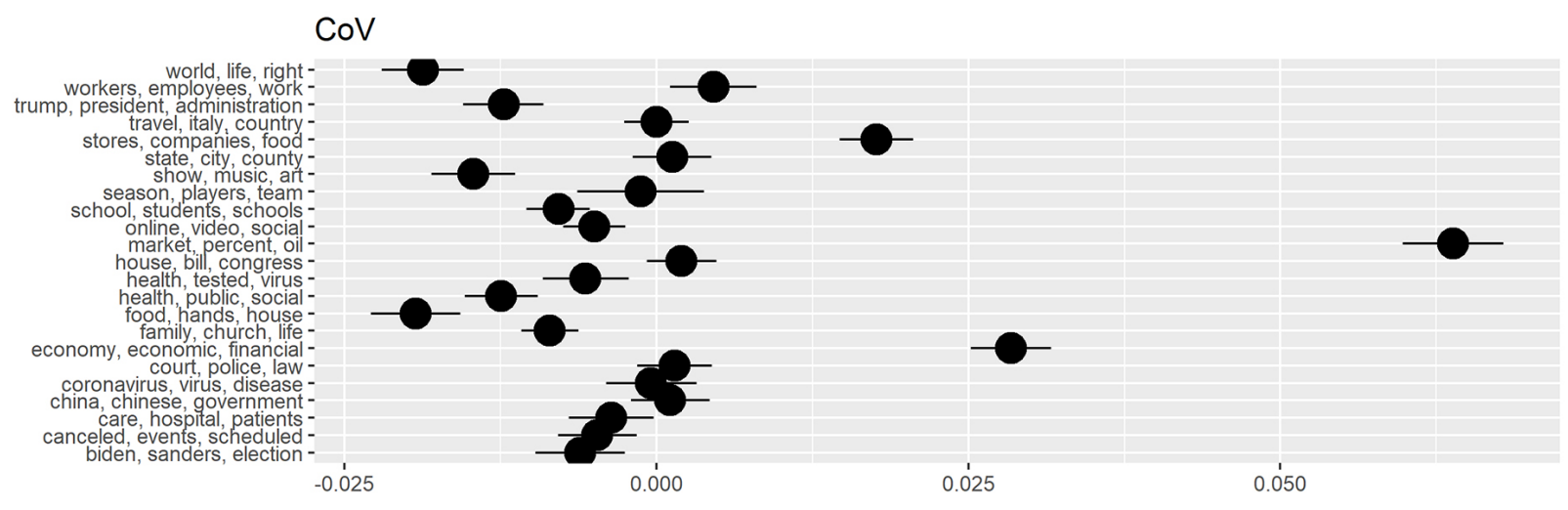

$\mathrm{H} 1 \mathrm{~N} 1$

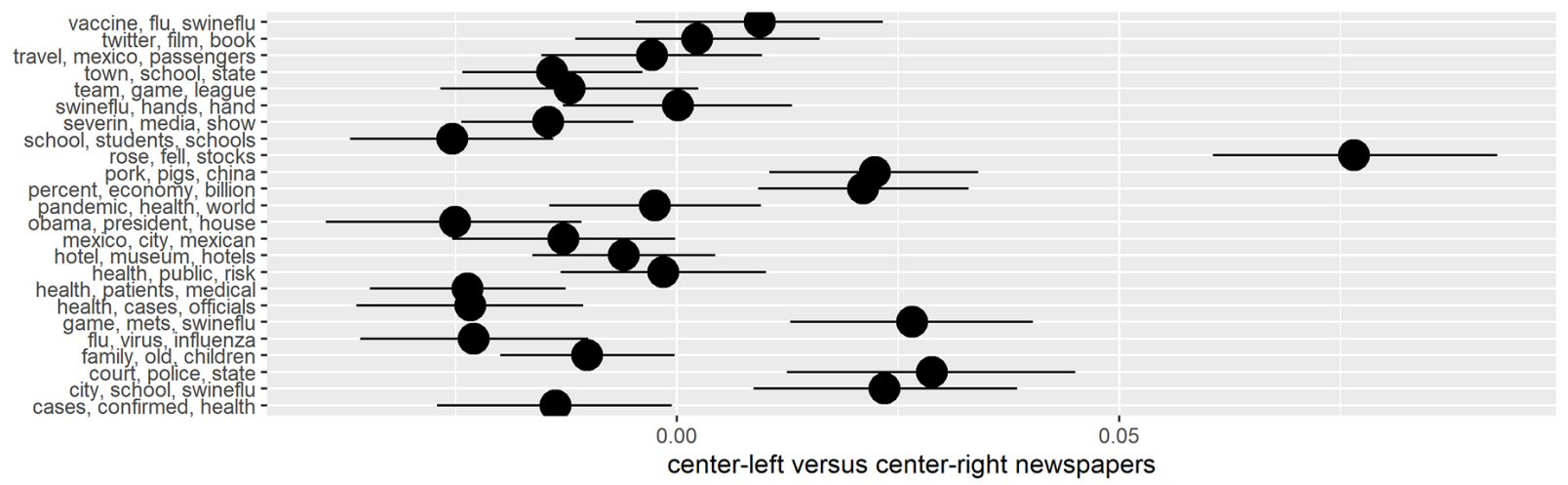

Figure 2. 


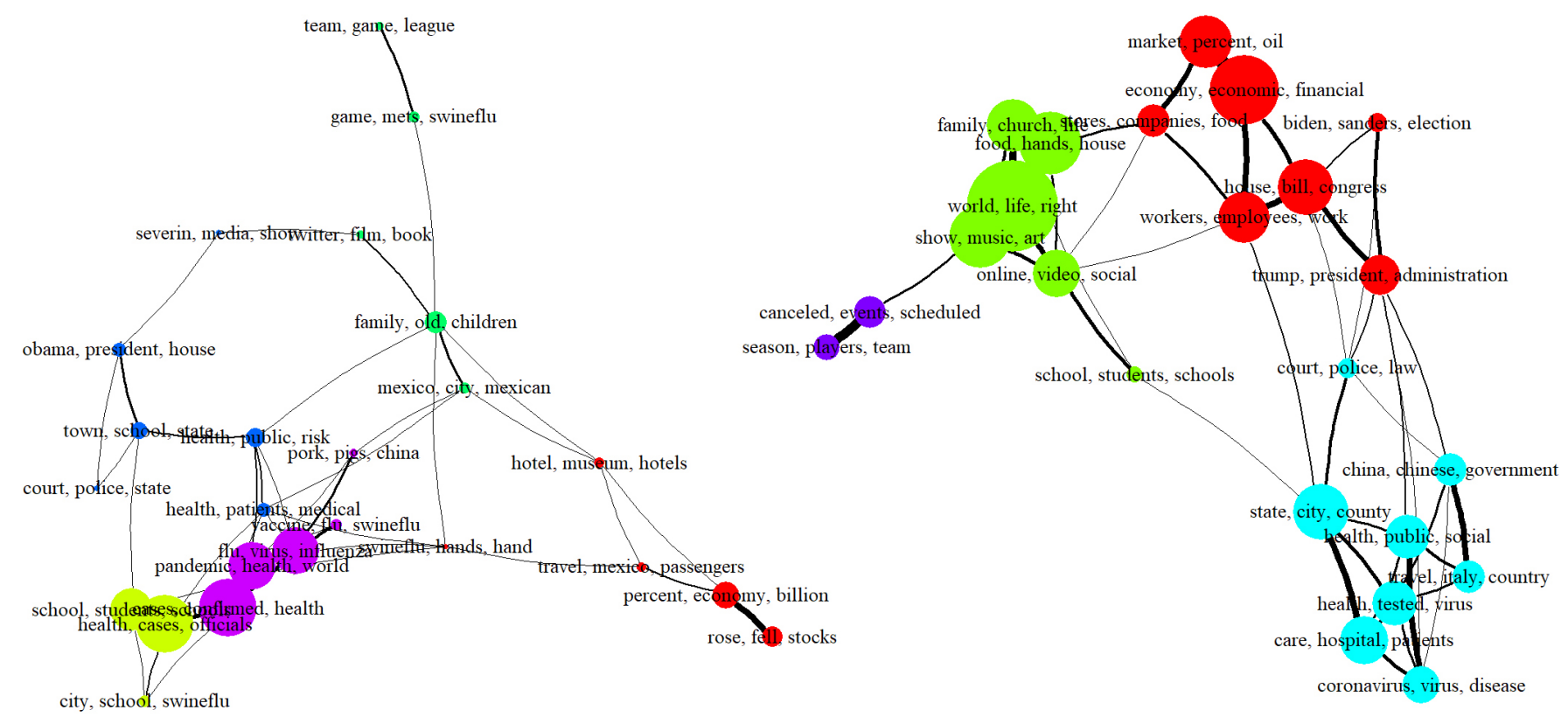

Figure 3.

these networks, highlighting respective community structures (represented by node colors). The topic network for the swine flu breaks down into five communities and the network for Covid-19 into four. The community structure for Covid-19 has a clearer semantic concept than is the case for the swine flu, as we can identify distinct discursive clusters that respectively relate to leisure time, sports, policy, and economics, as well as policy and public health. For the swine flu, we identify clusters that similarly deal with leisure time and sports, as well as policy and public health. In addition, we find a cluster that merges economics with travel and culture, a cluster on influenza pandemics and global public health, and one on schools. While community detection thus highlights significant similarities, the relatively low number of articles in the swine flu corpus translates into an unfavorable signal-to-noise ratio when analyzing the community structure in the respective topic network.

The similarities in network topology become more evident once we compare various key indicators: average clustering coefficients (the degree to which a given node forms closed triplets with the nodes in its neighborhood); average strength (the summed weights of all edges incidental to a given node); average node betweenness (the degree to which a node lies on the shortest paths through a network and thus serves as a mediator); strength centralization (total network centralization as calculated on edge weights); and betweenness centralization (total network centralization as calculated via edge betweenness). Figure 4 shows that, for four out of those five 
measurements, differences between Covid-19 and the swine flu are marginal: in each network, nodes have, on average, comparable probabilities of forming closed triplets, as well as similar betweenness. For both strength and betweenness, network centralization is highly similar as well. Only for average node strength do we see a notable difference, with the correlations among the topics of the Covid-19 corpus generally being stronger than is the case for the swine flu corpus.

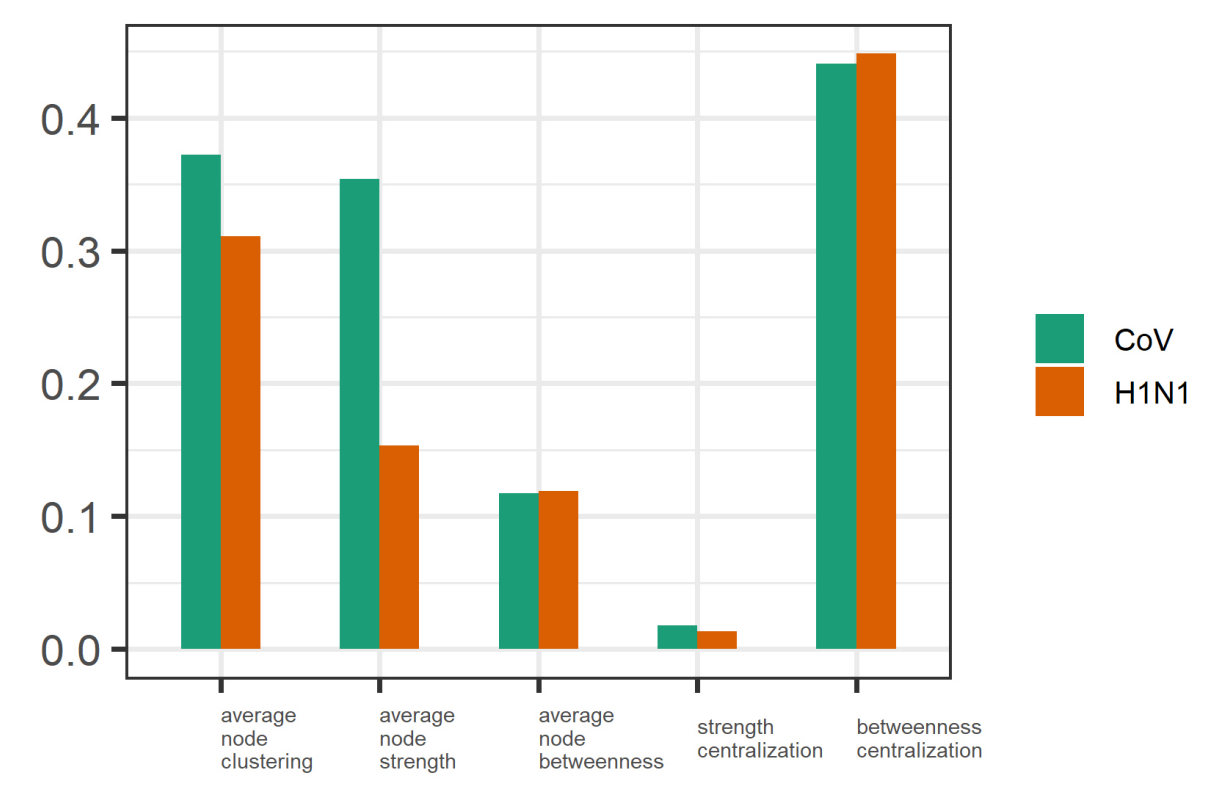

Figure 4.

Overall, our analysis thus highlights some differences in the news media representation of the two pandemics. The distribution of document-topic loadings is somewhat more skewed for the swine flu than for Covid-19; the community structure of the former has a more ambiguous semantic concept than is the case for the latter, and Covid-19 sees stronger linkages between the different discursive elements. At the same time, our analysis also shows strong similarities in content, left-right framing, and various measurements related to node clustering and centrality, and total network centralization. These results can indeed be interpreted in different ways, considering that some differences (notably for the analysis of community structure) likely derive from the relatively low amount of newspaper articles available for the swine flu case.

\section{Discussion}

The comparison of the semantic networks, understood as iconic models, resulting from topic models of the two datasets reveals several similarities but also some differences 
A Semiotic Comparison of Mass Media Representations of the Swine Flu and Covid-19 Pandemics: Observing Narcissus Narcosis

between how American newspapers reported on the H1N1 and the Covid-19 pandemics during periods when these two were broadly comparable. We argue that the particular structure of semantic similarities and discursive differences between these models indicates elements of a Narcissus narcosis phenomenon displayed by newspapers' reporting. It would be incorrect to claim that the models reveal merely an overall similarity or difference. Instead, in the broader picture of the mediascape and in light of the specific media changes that occurred in the meantime, the models point to a disguised (consciously or not) social change. To put it more simply, the discourse changed, along with the media, but as the semantics of newspaper articles broadly remain the same, it may give the impression that nothing (or not much) changed. The main similarities that we note consist in: (1) the absence of affective polarization according to ideology, (2) the presence of the same clusters of topics, and (3) similarities in network topology.

First, the similar left-right document loadings show that newspapers remained as polarizing during Covid-19 as they tended to be during H1N1 (see Figures 1 and 2 , above). This is a relevant finding because the overall discourse changed. Recently, other media, particularly social media, display a much more stringent affective polarization in American politics (e.g., Bail et al. 2018; Stjernfelt and Laurizen 2020: 40).

Second, identifying many of the same clusters in both models (see Figure 3, above) means that newspapers approach the same topics in both pandemic contexts, tending to convey the same type of information. In the spirit of Peirce's iconicity-based graph theory (i.e., Existential Graphs), which lies at the foundation of the modeling concept we adopt, these co-localizations (see also Stjernfelt 2019) of topics imply a relation of a subject-predicate type. As each model exhibits the same propositions, we infer that these newspapers have been conveying the same kind of information about the same subjects for each pandemic. Such clusters are leisure time and sports, policy and public health, travel and culture, pandemics, and public health and schools.

Third, we note that the topologies of the two networks are remarkably similar, as indicated by several critical indicators (see Figure 4 above).

\section{Conclusions}

Comparing the swine flu and Covid-19 pandemics offers insights into the social impacts of the recent rise of social media. We comparatively interpreted models of newspaper corpora before and after the mainstreaming of digital social media. Of particular importance is that social media forces newspapers to change as a medium by collapsing the media content producer/consumer dichotomy of broadcasting. Social media enable any consumer, with however a limited digital literacy, to be(come) a content creator, which also means that they are characterized by a virality that renders content creators 
too little control over the circulation and distortion of their content (Marwick and boyd 2010; Mills 2012). As such, information publicly conveyed by medical professionals about Covid-19 can reach various publics in variously distorted forms through a variety of viral channels. At the time of the swine flu pandemic, spiraling wrong information about a pandemic into a full-blown infodemic was not yet possible. Therefore, comparing the media coverage of these two pandemics offers an insight into this media change that is otherwise difficult to perceive while living it, as we find ourselves in, using McLuhan's (1994 [1964]: 55) celebrated expression, a "Narcissus-narcosis." In brief, newspapers, the older medium, manifest a numbness as necessary to settle in the new extension of humans onto social media. As McLuhan's concept is philosophically broad and, to some extent, speculative, it cannot be pinned down with precision by a chunk of data.

Nevertheless, we argue that this concept is descriptive of the conclusions drawn from our comparison of data models. The comparison does not merely indicate that a specific change took place or not. The relatively subtle argument to which the comparison leads is that by stubbornly reporting in the same rhetoric before and after a significant media and discursive change, newspapers are behaving like a 'counter-irritant,' an anesthetic meant to render the ongoing media change more bearable.

This masks the fact that digitalization is manifest, among other ways, by social media taking the place of older media as the primary source of news. It is only very recently that, at least in the US, social media toppled television as the primary source for news (see Shearer 2018; Mitchell and Shearer 2020). In this regard, this media change is different from the emergence of, for example, radio and TV broadcasting. The latter has been the primary source of news for a long time, but it did not replace or alter newspapers from becoming a different medium than they used to be. The newspaper medium has probably undergone some adaptations once it had to run alongside, say, radio, but the newspaper could not altogether move into a radio format. By contrast, newspapers now have to interact with their publics through their channels on social networking sites. The interpretation of current broadcasted information should not overlook that digitalization does not consist only in the emergence of new media, but it also implies the digitalization of older media. We observe Narcissus narcosis statistically, as the stability in the correlation of ideology and topics across two different discourses. Simplifying, while newspapers, too, illustrate that the new media context changed the discourse on pandemics altogether, they still address the same matters as before this change. One possible reason the newspaper medium, in particular, acts as counter-irritant consists of the characteristic of articles to be assigned distinctive authorship. They lack the virality and anonymity that are intrinsic to social media dynamics. At the same time, the virality of social media likely influences the content of newspapers. In McLuhan's terms, newspapers (or, rather, their publics) are experiencing a "numbness" that allows ignoring or, instead, renders invisible the amputation necessary for the extension. 
A Semiotic Comparison of Mass Media Representations of the Swine Flu and Covid-19 Pandemics: Observing Narcissus Narcosis

To conclude, while newspapers have been the primary source for news in the modern age, digitalization pushes them to change style and, arguably, their overall mediality (e.g., Hong 2012; Ju et al. 2014). To survive, newspapers now have to meet the requirements of the public accustomed to the dynamics of social media. As such, newspapers seek to expand their social media networks, which become integrated, not without their agency, however limited, in virulent social networks.

Comparing representations of the two pandemics in newspapers is insightful because newspapers are well-established communication channels with an assumed conventional type of authority. Traditionally, newspapers are perceived as accountable (different from reliable), without deceivers intrinsic to their infrastructure, as is the case of new media (see Genosko 2012). The stubborn rhetoric of newspapers proves a strong anesthetic, obscuring their integration into the viral dynamics of social media networks. Consuming newspaper content under this assumption displays a lack of media literacy, as newspapers tend to assimilate the discourses circulating in new media. The virality of social networking sites cannot be comprehended by old models of communication consisting of unidirectional messages originating in an identifiable source, such as many oversimplified uses of the classic Shannon and Weaver information-based model (1964; for similar criticism, see Kress 2010: 36; Genosko 2012: 30-31). Neither can such models describe the work of newspapers anymore, as these adapt to and are assimilated into digital networks through a process of "viral transduction" (Genosko 2012: 14). Our observation that, at least in respect to the swine flu and the Covid-19 pandemics, we are currently undergoing a Narcissus narcosis, contributes to the argument that, given the virality of media networks, linear communication models are insufficient for cultural criticism and the analysis of social representations. We presented one alternative consisting of a semiotic outlook on media content organized in topic models and semantic networks.

\section{FUNDING}

This article was supported by grant no. PP59/2016 ("A Topic-Network Approach to the Mass Media Representation of Pandemic Threats") of the Special Research, Development and Innovation Fund of Kaunas University of Technology and by the Cognitive Linguistics and Semiotics Research Group of RWTH Aachen University, which hosted Florian Rabitz as a visiting researcher during March-April 2021. 


\section{References}

Andersen, Martin 2020. Early evidence on social distancing in response to COVID-19 in the United States. Available at SSRN: https://ssrn.com/abstract=3569368 or http: / / dx.doi.org/10.2139/ssrn.3569368

Au, Hubert, Jonathan Bright and Philip N. Howard.=2020. Social Media Misinformation about the WHO. COMPROP Coronavirus Misinformation Weekly Briefing 27.04.2020, 1-3.

Bail, Christopher A., Lisa P. Argyleb, Taylor W. Brown, John P. Bumpus, Haohan Chen, M. B. Fallin Hunzakerd, Jaemin Lee, Marcus Manna, Friedolin Merhouta, and Alexander Volfovsky 2018. Exposure to opposing views on social media can increase political polarization. Proceedings of the National Academy of Sciences of the United States of America 115(37): 9216-9221.

Bright, Johnathan, Hubert Au, Hannah Bailey, Mona Elswah, Marcel Schliebs, Nahema Marchal, Christian Schwieter, Katarina Rebello and Philip N. Howard. 2020. Coronavirus Coverage by State-Backed English-Language News Sources: Understanding Chinese, Iranian, Russian and Turkish Government Media. COMPROP Data Memo 2: 1-7.

Canter, Lily 2013. The interactive spectrum: The use of social media in UK regional newspapers. Convergence: The International Journal of Research into New Media Technologies 19(4): 472-495.

Centers for Disease Control and Prevention 2019. 2009 H1N1 Pandemic Timeline. May 8, 2019, retrieved from https:/ / www.cdc.gov/flu/pandemic-resources/2009-pandemic-timeline.html, accessed on August 5, 2020.

Centers for Disease Control and Prevention 2021. COVID Data Tracker. Retrieved from https: / / covid.cdc.gov/ covid-data-tracker/\#datatracker-home, accessed on March 26, 2021.

Cinelli, M., W. Quattrociocchi, A. Galeazzi, C.M. Valensise, E. Brugnoli, A.L.Schmidt, F. Zola, and A. Scala 2020. The COVID-19 social media infodemic. Nature 10: 16598.

Chaiuk, Tetyana A., and Olha V. Dunaievska 2020. Producing the Fear Culture in Media: An Examination on Coronavirus Discourse. Journal of History Culture and Art Research 9(2): 184-194.

Ciula, Arianna, and Eide Øyvind 2017. Modelling in digital humanities: Signs in context. Digital Scholarship in the Humanities 32(1): i33-i46.

Danto, Arthur 2007. Narration and Knowledge. New York: Columbia University Press.

DiMaggio, P., M. Nag, and D. Blei 2013. Exploiting Affinities between Topic Modeling and the Sociological Perspective on Culture: Application to Newspaper Coverage of US Government Arts Funding. Poetics 41(6): 570 - 606.

Garfin, D.R., R.C. Silver, and E.A. Holman 2020. The Novel Coronavirus (COVID-2019) Outbreak: Amplification of Public Health Consequences by Media Exposure. Health Psychology 39(5): 355-357. 
A Semiotic Comparison of Mass Media Representations of the Swine Flu and Covid-19 Pandemics: Observing Narcissus Narcosis

Genosko, Gary 2012. Remodelling Communication: From WWII to the WWW. Toronto: University of Toronto Press.

Goodall, C., J. Sabo, R. Cline, and N. Egbert 2012. Threat, Efficacy, and Uncertainty in the First 5 Months of National Print and Electronic News Coverage of the H1N1 Virus. Journal of Health Communication 17: 338 - 355.

Hong, Sounman 2012. Online news on Twitter: Newspapers' social media adoption and their online readership. Information Economics and Policy 24(1): 69 -74.

Hua, Jinling and Rajib Shaw 2020. Corona Virus (COVID-19) “Infodemic” and Emerging Issues through a Data Lens: The Case of China. Int. J. Environ. Res. Public Health 17:1-12.

Kress, Gunther 2010. Multimodality: A social semiotic approach to contemporary communication. New York: Routledge.

Leeuwen, Theo van 2005. Introducing social semiotics. New York: Routledge.

Litt, Eden 2012. Knock, knock. Who's there? The imagined audience. Journal of Broadcasting E Electronic Media 56(3): 330-345.

Litt, E., and E. Hargittai 2016. The imagined audience on social networking sites. Social Media + Society 2(1): 1-6.

Jo, W., J. Lee, J. Park, and Y. Kim 2020. Online Information Exchange and Anxiety Spread in the Early Stage of the Novel Coronavirus (COVID-19) Outbreak in South Korea: Structural Topic Model and Network Analysis. J Med Internet Res 22(6): 1 - 20.

Ju, Alice, Sun Ho Jeung, and Hsiang Iris Chyi 2014. Will social media save newspapers? Examining the effectiveness of Facebook and Twitter as news platforms. Journalism Practice 8(1): 1-17.

Klebanov, B. B., D. Diermeier, and E. Beigman 2008. Automatic Annotation of Semantic Fields for Political Science Research. Journal of Information Technology \& Politics 5(1): 95-120.

Klemm, C., E. Das, and T. Hartmann 2016. Swine flu and hype: a systematic review of media dramatization of the H1N1 influenza pandemic. Journal of Risk Research 19(1): 1- 20.

Kralemann, Björn and Claas Lattmann 2013. Models as icons: modeling models in the semiotic framework of Peirce's theory of signs. Synthese 190: 3397-3420.

Krishnatray, Pradeep, and Rahul Gadekar 2014. Construction of death in H1N1 news in The Times of India. Journalism 15(6): 731-753.

Marwick, Alice E. and danah boyd 2012. I tweet honestly, I tweet passionately: Twitter users, context collapse, and the imagined audience. New media E society 13(1): 113-133.

Mills, Adam J. 2012. Virality in social media: the SPIN framework. Journal of Public Affairs 12(2): 162-169.

McLuhan, Marshall 1994 [1964]. Understanding Media: The Extensions of Man. Intr. by Lewis H. Lapham. Cambridge, Massachusetts: The MIT Press. 
Merchant, Raina M. and Nicole Lurie 2020. Social media and emergency preparedness in response to novel coronavirus. Journal of the American Medical Association 323(20): 2011-2012. CP = Peirce, Charles S. 1958 [1931-1958]. The Collected Papers of Charles Sanders Peirce. Eds. Hartshorne, Charles; Weiss, Paul, Arthur W. Cambridge: Belknap.

Pew Research Center 2021. Social Media Fact Sheet. Pew Research Center. Available at https: / / www.pewresearch.org/internet/ fact-sheet/ social-media/. Accessed July 7, 2021.

Mitchel, A. and E. Shearer 2020. News Use Across Social Media Platforms in 2020. Pew Research Center, January 12, 2021.

Rabitz, F., A. Olteanu, J. Jurkevičienè, and A. Budžytè 2019. The case for a semiotic method in Earth system science: Semantic networks of environmental research. Sign Systems Studies 47(3/4): 552-589.

Rabitz, F., A. Olteanu, J. Jurkevičienė, and A. Budžytė 2021. A Topic Network Analysis of the System Turn in the Environmental Sciences. Scientometrics 126(3): 2107-2140.

Roberts, M. E., B. M. Stewart, and D. Tingley 2016. Navigating the Local Modes of Big Data. In: Computational social science: Discovery and Prediction. New York: Cambridge University Press, 51-97.

Shannon, Claude E., and Warren Weaver 1964. The Mathematical Theory of Communication. Urbana: University of Illinois Press.

Shearer, E. 2018. Social media outpaces print newspapers in the U.S. as a news source. Pew Research Center, December 10, 2018.

Shearer, E. 2021. More than eight-in-ten Americans get news from digital devices. Pew Research Center, January 12, 2021.

Stjernfelt, Frederik 2007. Diagrammatology: An investigation on the borderlines of phenomenology, ontology, and semiotics. Dordrecht: Springer.

Stjernfelt, Frederik 2019. Co-localization as the Syntax of Multimodal Propositions: An Amazing Peircean Idea and Some Implications for the Semiotics of Truth. In: T. Jappy (ed.) The Bloomsbury Companion to Contemporary Peircean Semiotics. London: Bloomsbury, 419-458.

Stjernfelt, Frederik and Anne Mette Lauritzen 2020. Your post has been removed: Tech giants and freedom of speech. Cham: Springer.

World Health Organization 2020a. World Health Statistics 2020: A Visual Summary. Retrieved from https: / / www.who.int/ data/gho/whs-2020-visual-summary, accessed on July 28, 2020.

World Health Organization 2020b. Q\&A: Influenza and COVID-19 - similarities and differences. Published March 17, retrieved from https://www.who.int/emergencies / diseases / novel-coronavirus-2019 / question-and-answers-hub / q-a-detail / q-a-similarities-and-differences-covid-19-and-influenza?gclid=CjwKCAjwsan5BRAOEiwALzomX_1EjpxPJK0BGAgMUk77sb-X2pVa5NobwpqN72r39uyxLwEetfDSKhoCwkcQAvD_BwE, accessed on August 5, 2020. 
A Semiotic Comparison of Mass Media Representations of the Swine Flu and Covid-19 Pandemics: Observing Narcissus Narcosis

World Health Organization 2020c. Countering misinformation about COVID-19: A joint campaign with the Government of the United Kingdom. Published May 11, retrieved from https: / / www.who.int/ news-room/feature-stories/ detail/ countering-misinformation-about-covid-19, accessed March 26, 2021.

World Health Organization 2021. Countering health misinformation in the comments section, with \#iamhere! Published February 17, retrieved from https://www. who.int/news-room / events / detail / 2021 / 02/24/ default-calendar/ countering-health-misinformation-in-the-comments-section-with-iamhere!, accessed on March 26, 2021.

\section{AUTHOR}

Alin Olteanu Researcher and Publications Coordinator at the KHK Cultures of Research center of RWTH Aachen University and Senior Researcher at the Kaunas University of Technology, Lithuania.

\section{AUTHOR}

Florian Rabitz Chief researcher at the Civil Society and Sustainability Research Group, Kaunas University of Technology, Lithuania.

\section{AUTHOR}

Augustè Nalivaikè Junior researcher at the Civil Society and Sustainability Research Group and Ph.D. candidate at the Kaunas University of Technology, Lithuania. 